Received: 18.10 .2018

Revised: 26.11 .2018

Accepted: 30.11 .2018

DOI: $10.17804 / 2410-9908.2018 .6 .173-183$

\title{
DETERMINATION OF THERMAL EFFECTS OF DIFFUSIONLESS PHASE TRANSFORMATIONS IN LOW-CARBON LOW-ALLOY STEELS AT HIGH COOLING RATES
}

\author{
I. A. Artemiev ${ }^{1, \text { a) }}$, M. L. Krasnov ${ }^{2, \text { b) }}$, G. M. Rusakov ${ }^{3,4, c)}$, and S. V. Danilov ${ }^{4, d}{ }^{*}$ \\ ${ }^{1}$ Ausferr Research and Technology Center, 18 Gorkogo St., Magnitogorsk, 455000, Russian Federation \\ ${ }^{2}$ Magnitogorsk Iron and Steel Works OJSC, 93 Kirova St., Magnitogorsk, 455000, Russian Federation \\ ${ }^{3}$ M. N. Miheev Institute of Metal Physics, Ural Branch of the Russian Academy of Sciences, \\ 18 S. Kovalevskoy St., Ekaterinburg, 620990, Russian Federation \\ ${ }^{4}$ B. N. Yeltsin Ural Federal University, 19 Mira St., Ekaterinburg, 620002, Russian Federation
}

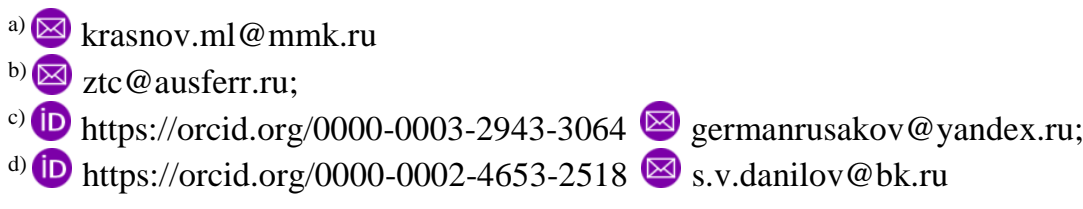

*Corresponding author. E-mail: s.v.danilov@bk.ru

Address for correspondence: ul. Mira, 19, Ekaterinburg, 620002, Russian Federation

Tel.: +7 (343) 3754803

We have designed an original laboratory bench allowing one to model technological rates of cooling of hot-rolled sheets in the process of controlled thermomechanical processing. The bench consists of a heating furnace with a mechanism for sample extraction and fixation, a temperature measurement unit comprising three pyrometers, an air-cooling unit with a pneumatic system, a unit for management and information recording. The sample cooling rate is controlled through sample thickness and pressure in the air cooling system. We use samples cut from an industrial sheet of the 06G2MB-type low-carbon low-alloy pipe steel, which is intended for production of large pipes of the X80 strength class. We have obtained time dependencies of sample temperatures at cooling rates ranging from 50 to $700{ }^{\circ} \mathrm{C} / \mathrm{s}$. The heat effect of bainitic transformation is equal to $120 \pm 5 \mathrm{~kJ} / \mathrm{kg}$, this being twice the thermal effect of martensitic transformation for low-carbon steels.

Keywords: thermal effect of phase transition, pipeline steel, controlled thermomechanical processing, cooling rate, martensitic transformation, bainitic transformation.

\section{Acknowledgment}

The study was performed on the equipment from the Ausferr JSC within the framework of the state assignment from the Federal Agency for Scientific Organizations of the Russian Federation, theme Structure, registration number 0120146333. We express our gratitude for the assistance to the Program of Support Rendered to the Leading Universities of the Russian Federation in Order to Improve Their Competitiveness, No. 211, of the Government of the Russian Federation No. 02.A03.21.0006. We are also grateful to the MISW PJSC for their support and instrumentality in the organization of the research.

\section{References}

1. Sung H.K., Shin S.Y., Hwang B., Lee C.G., Kim N.J., Lee S. Effects of carbon equivalent and cooling rate on tensile and Charpy impact properties of high-strength bainitic steels. Materials Science and Engineering A, 2011, vol. 530, pp. 530-538. DOI: 10.1016/j.msea.2011.10.015. 
2. Liang X.J., Hua M.J., Garcia C.I., Deardo A.J. The thermomechanical controlled processing of high-strength steel plate: A New View of toughness based on modern metallography. Materials Science Forum, 2013, vol. 762, pp. 38-46. DOI: 10.4028/www.scientific.net/MSF.762.38.

3. Goli-Oglu E.A., Éfron L.I., Morozov Yu.D. Effect of deformation regime in main stages of controlled rolling on pipe steel microstructure. Metal Science and Heat Treatment, 2013, vol. 55, pp. 294-297. DOI: 10.1007/s11041-013-9622-8.

4. Shigeru E., Naoki N. Development of Thermo-Mechanical Control Process (TMCP) and high performance steel in JFE Steel. JFE Technical Report, 2015, no. 20, pp. 1-7.

5. Lobanov M.L., Borodina M.D., Danilov S.V., Pyshmintsev I.Y., Struin A.O. Texture Inheritance on Phase Transition in Low-Carbon, Low-Alloy Pipe Steel after Thermomechanical Controlled Processing. Steel in Translation, 2017, vol. 47, pp. 710-716. DOI: 10.3103/S0967091217110055.

6. Pyshmintsev I.Y., Struin A.O., Gervasyev A.M., Lobanov M.L., Rusakov G.M., Danilov S.V., Arabey A.B. Effect of bainite crystallographic texture on failure of pipe steel sheets made by controlled thermomechanical treatment. Metallurgist, 2016, vol. 60, pp. 405-412. DOI: $10.1007 / \mathrm{s} 11015-016-0306-7$.

7. Sabirov I., De Diego-Calderón I., Molina-Aldareguia J. M., Föjer C., Thiessen R., Petrov R.H. Microstructural design in quenched and partitioned (Q\&P) steels to improve their fracture properties. Materials Science and Engineering A, 2016, vol. 657, pp. 136-146. DOI: 10.1016/j.msea.2016.01.011.

8. Gong W., Tomota Y., Paradowska A.M., Kelleher J.F., Zhang S.Y. Effects of ausforming temperature on bainite transformation, microstructure and variant selection in nanobainite steel. Acta Materialia, 2013, vol. 61, pp. 4142-4154. DOI: 10.1016/j.actamat.2013.03.041.

9. Hulka K., Peters P., Haisterkamp F. Trends in the Development of Large-Diameter Pipe Steels. Steel in Translation, 1997, vol. 27, no. 10, pp. 64-70. (In Russian).

10. Lobanov M.L., Yurovskikh A.S., Kardonina N.I., Rusakov G.M. Metody issledovaniya tekstur v materialakh: uchebnoe posobie [Methods of Study of Textures in Materials]. Izd. Uralsk. Univ. Publ., Ekaterinburg, 2014, 115 p. (In Russian).

11. Lobanov M.L., Rusakov G.M., Redikul'tsev A.A., Belikov S.V., Karabanalov M.S., Struina E.R., Gervas'ev A.M. Investigation of Special Misorientations in Lath Martensite of Low-Carbon Steel Using the Method of Orientation Microscopy. The Physics of Metals and Metallography, 2016, vol. 117, pp. 254-259. DOI: 10.1134/S0031918X1603008X.

12. Lobanov M.L., Rusakov G.M., Urtsev V.N., Krasnov M.L., Mokshin E.D., Shmakov A.V., Platov S.I. Thermal effect of bainitic transformation in tube steels during accelerated cooling. Letters on Materials, 2018, vol. 8 (3), pp. 246-251. DOI: 10.22226/2410-3535-2018-3-246-251.

13. Ivanov D.A., Kuvayev N.V., Kuvayeva T.V. Calculation of the heat capacity of low-carbon low-alloy steel in modeling non-isothermal phase transformations. Teoriya i Praktika Metallurgii, 2010, no. 1-2, pp. 43-48. (In Russian).

14. Sufiyanova G. I., Spivak L. V. Fazovye prevrashcheniya nizkouglerodistykh martensitnykh stalyakh. In: Fizika dlya Permskogo kraya: Conference proceedings, Perm, 2016, Permskiy Gos. Nats. Issled. Un-t Publ., 2016, pp. 37-39. (In Russian).

15. Bhadeshia H.K.D.H. Martensite and bainite in steels: Transformation mechanism \& mechanical properties. Journal De Physique IV : JP, 1997, vol. 7 (5), pp. C5-367-C5-376.

16. Gol'dshtein M.I., Popov V.V. Rastvorimost' faz vnedreniya pri termicheskoi obrabotke stali [Solubility of Interstitial Phases in the Heat Treatment of Steel]. Moscow, Metallurgiya Publ., 1989 (In Russian). 
Подана в журнал: 18.10 .2018

УДК 669.15-194.2:53.06

DOI: $10.17804 / 2410-9908.2018 .6 .173-183$

\title{
ОПРЕДЕЛЕНИЕ ТЕПЛОВЫХ ЭФФЕКТОВ СДВИГОВЫХ ФАЗОВЫХ ПРЕВРАЩЕНИЙ В МАЛОУГЛЕРОДИСТЫХ НИЗКОЛЕГИРОВАННЫХ СТАЛЯХ ПРИ БОЛЬШИХ СКОРОСТЯХ ОХЛАЖДЕНИЯ
}

\author{
И. А. Артемьев ${ }^{1, \text { a) }}$, М. Л. Краснов ${ }^{2, \text { б) }}$, Г. М. Русаков ${ }^{3,4, \text { в) }}$ С. В. Данилов ${ }^{4, \text { г)* }}$ \\ ${ }^{1}$ ИТЦ «Аусферр», ул. Горького 18, 455000, Магнитогорск, Российская Федераџия \\ ${ }^{2}$ Магнитогорский металлургический комбинат, ул. Кирова, 93, 455000, Магнитогорск, Российская Федерация \\ ${ }^{3}$ Институт физики металлов имени М.Н. Михеева Уральского отделения РАН, \\ ул. С. Ковалевской 18, 620990, Екатеринбург, Российская Федерация \\ ${ }^{4}$ Уральский федеральный университет имени первого Президента России Б.Н. Ельиина, \\ ул. Мира 19, 620002, Екатеринбург, Российская Федеращия \\ г) \\ krasnov.ml@mmk.ru \\ ztc@ausferr.ru; \\ B) https://orcid.org/0000-0003-2943-3064 \\ iD https://orcid.org/0000-0002-4653-2518 \\ germanrusakov@yandex.ru; \\ s.v.danilov@bk.ru \\ *Ответственный автор. Электронная почта: s.v.danilov@ bk.ru \\ Адрес для переписки: ул. Мира 19, 620002, Екатеринбург, Российская Федерация \\ Тел.: +7 (343) 375-48-03
}

Разработан оригинальный лабораторный стенд, позволяющий моделировать технологические скорости охлаждения горячекатаных листов в процессе контролируемой термомеханической обработки. Стенд состоит из нагревательной печи с механизмом извлечения и фиксации образцов, блока измерения температуры, включающего три пирометра, узла воздушного охлаждения с пневмосистемой, узла управления и регистрации информации. Скорость охлаждения образцов регулировали толщиной образцов и давлением в системе воздушного охлаждения. В качестве материалов для исследований использовали образцы, вырезанные из промышленного листа малоуглеродистой низколегированной трубной стали типа 06Г2МБ, предназначенного для производства труб большого диаметра класса прочности Х80. Были получены зависимости температуры образцов от времени при скоростях охлаждения 50-700 ${ }^{\circ} \mathrm{C} / \mathrm{c}$. Величина теплового эффекта бейнитного превращения оказалась равной $120 \pm 5$ кДж/кг, что в два раза превышает тепловой эффект мартенситного превращения для низкоуглеродистых сталей.

Ключевые слова: тепловой эффект фазового превращения, трубные стали, контролируемая термомеханическая обработка (ТМСР), мартенситное превращение, бейнитное превращение.

\section{1. Введение}

Современный уровень производства трубных изделий ответственного назначения с заданными функциональными свойствами и прогнозируемыми структурами, требует управления параметрами технологических процессов в соответствии с кинетикой протекающих в материале физических процессов [1-5]. Важнейшими характеристиками процессов структурных и фазовых превращений, протекающих в материале при его обработке, являются величины их тепловых эффектов. Удельные величины тепловых эффектов наряду с технологическими параметрами определяют температуру материала в различные моменты его продвижения по технологической цепочке, и в итоге могут существенно влиять на структуру и свойства материала или изделия [1, 6-8]. 
Значимой проблемой при прецизионном определении тепловых эффектов превращений с использованием лабораторных калориметров являются сравнительно медленные скорости охлаждения образцов, т. е. скорости, существенно отличные от параметров, реализующихся в условиях промышленного производства. Очевидно, что различия в лабораторных и промышленных скоростях охлаждения приводят как к смещению температурных интервалов превращений, так и к искажению величин тепловых эффектов последних.

Цель работы - определение величин тепловых эффектов бейнитного и мартенситного превращений в малоуглеродистой низколегированной трубной стали, используемой при TMCP (Thermo-Mechanical Controlled Processing), необходимых для моделирования и, соответственно, управления процессами контролируемой термомеханической обработки на современных прокатных станах.

\section{2. Материалы и методика}

Для моделирования кинетики теплофизических процессов, протекающих в сталях при скоростях охлаждения, воспроизводящих технологические параметры обработки (ТМСР), ИТЦ «Аусферр» разработал стенд (рис. 1), состоящий из нагревательной печи с механизмом извлечения и фиксации образцов, блока измерения температуры, узла воздушного охлаждения с пневмосистемой, узла управления и регистрации информации.

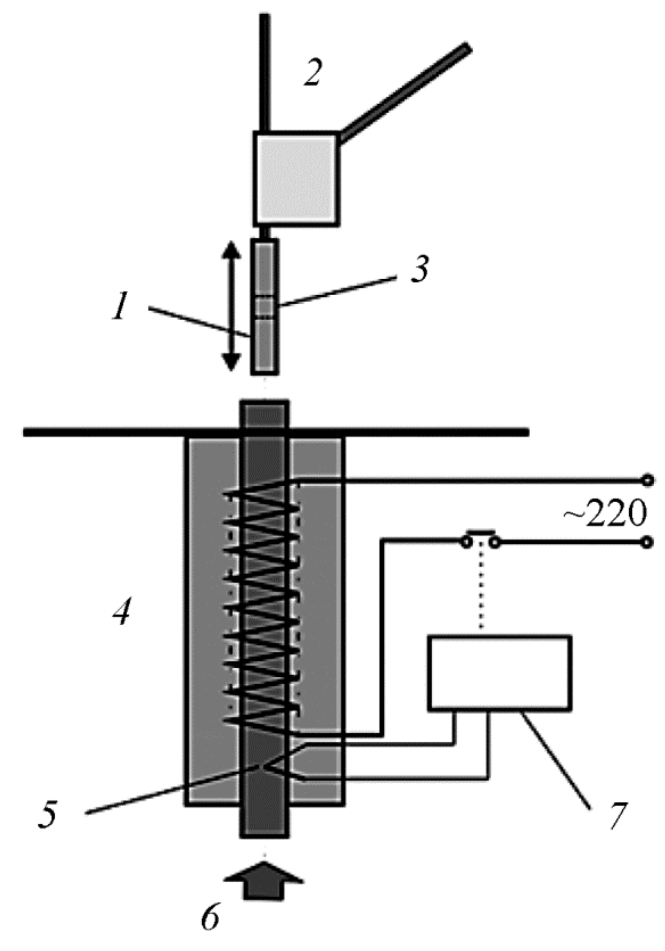

$a$

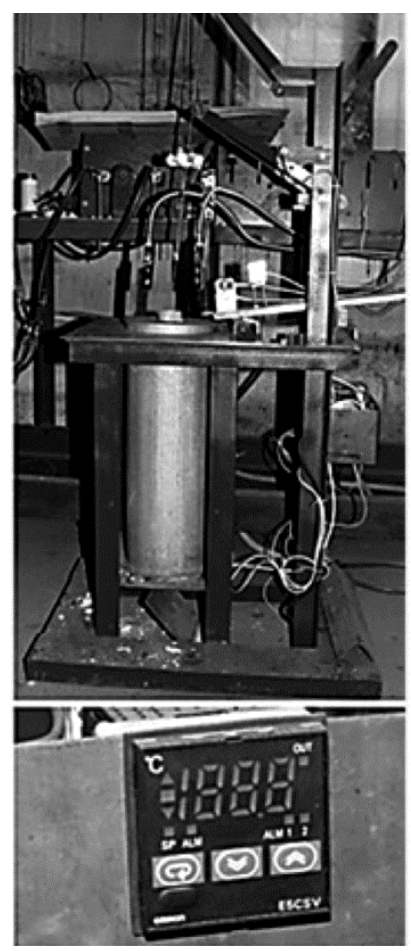

$\sigma$

Рис. 1. Установка для определения тепловых эффектов фазовых превращений при больших скоростях охлаждения: $a$ - схема; $\sigma$ - внешний вид; 1 - образец; 2 - механизм перемещения образца; 3 - область образца для измерений температуры; 4 - шахтная печь;

5 - термопара; 6 - подача аргона; 7 - контроллер печи

Вертикальная печь с цилиндрическим муфелем управляется независимым контроллером, поддерживающим заданную температуру. Для уменьшения окисления образцов в печь производится поддув аргона. Установка снабжена механизмом, позволяющим быстро извле- 
кать образец из печи и фиксировать его в рабочей зоне установки. Привод механизма - ручной, механизм снабжен концевым выключателем, запускающим процедуру регистрации.

Для измерения температуры применяются три пирометра. Два из них - Raytek Marathon FR1A - двухрежимные пирометры ближнего инфракрасного спектра, имеющие диапазон измерения 550-1100 ${ }^{\circ} \mathrm{C}$, третий - быстродействующий пирометр OPTRIS CTfast, работающий в среднем ИК-диапазоне. Диапазон измеряемых температур прибора -50-775 ${ }^{\circ} \mathrm{C}$. Перед измерением пирометры юстируются в точку, находящуюся в центре образца, извлеченного из печи. С этой же точкой совмещаются оси охлаждающих струй воздуха.

При пирометрических измерениях основная погрешность измерения определяется тем, что неизвестен коэффициент черноты диагностируемой поверхности. В применявшейся методике эта проблема решается тем, что один из пирометров FR1A работает в режиме спектральных отношений. Показания пирометра спектральных отношений, работающего на близких спектральных линиях, не зависят от коэффициента черноты. Это позволяет откалибровать показания двух оставшихся пирометров непосредственно в эксперименте, и, тем самым, реализовать измерения температуры с высокой точностью в диапазоне $50-1100{ }^{\circ} \mathrm{C}$.

Обдув образцов производится плоским потоком воздуха, направленным под углом 15-30 ${ }^{\circ}$ образцу для исключения теневых зон. Пневмосистема установки обеспечивает поддержание стабильного давления в магистралях в течение всего эксперимента. Принудительное охлаждение образцов производится с помощью блока сопел, имеющего 10 отверстий. Такая геометрия блока сопел позволяет получить однородный, близкий к плоскому, поток воздуха в зоне образца. Кроме блоков сопел пневмосистема стенда включает в себя компрессор с ресивером, редуктор, электромагнитный клапан, сенсоры для измерения давления.

Регистрация информации и управление установкой в процессе эксперимента проводится специализированным вычислительным узлом, работающим под управлением ОС реального времени. Основная экспериментальная информация - показания пирометров. Кроме того, контролируются давления в пневмосистеме и срабатывание концевого датчика. К каждому измерению система привязывает его время, что позволяет совмещать показания датчиков разных измерительных каналов.

В ходе эксперимента образец устанавливается в механизме перемещения и фиксации. С помощью узла юстирования все пирометры фокусируются в центральную точку поверхности образца. С этой же точкой совмещаются оси охлаждающих струй воздуха. Далее образец перемещается в печь, где нагревается до заданной температуры. После завершения выдержки происходит возвращение образца в первоначальное положение. Срабатывание концевых датчиков запускает процессы воздушного обдува и регистрации показаний пирометров.

В качестве материалов для исследований использовались образцы, вырезанные из промышленного листа малоуглеродистой низколегированной стали типа 06Г2МБ ( 0,05 мас. \% С; $\leq 2,0$ мас. $\% \mathrm{Mn} ; \sim 0,2$ мас. $\% \mathrm{Mo} ; \sim 0,05$ мас. $\% \mathrm{Nb}$, остальное железо и неизбежные примеси), предназначенного для производства труб большого диаметра классов прочности К60, К65. Образцы представляли собой пластины $20 \times 70$ мм, толщина которых варьировалась в диапазоне от 0,25 до 1,10 мм. Подобные размеры образцов обеспечивали практически одномерный теплообмен при процессах их нагрева или охлаждения.

Образцы нагревали в печи стенда до температуры аустенитизации $980{ }^{\circ} \mathrm{C}$ (без растворения карбидных фаз, выделившихся при промышленной ТМСР) [9] и выдерживали в течении 30 мин. Скорость охлаждения образцов регулировали толщиной образцов и давлением в системе воздушного охлаждения.

В результате экспериментов был получен набор образцов, охлажденных в температурном интервале от $980{ }^{\circ} \mathrm{C}$ до эффективной температуры начала фазовых превращений со скоростями $(d T / d t)$ в интервале $50-700{ }^{\circ} \mathrm{C} / \mathrm{c}$. По экспериментально полученным термограммам (рис. 2 a) определяли эффективные температуры начала и конца фазовых превращений, временные интервалы их реализации и тепловые эффекты. 
Структурные составляющие в охлажденных с различными скоростями образцах выявляли с использованием ориентационной микроскопии, основанной на дифракции обратно рассеянных электронов (EBSD) [10], по спектрам межкристаллитных границ, включая специальные $[5,11]$. На образцах также приготовляли металлографические шлифы для исследования микроструктуры и локальной текстуры. Электронно-микроскопическое исследование структуры проводили на растровом микроскопе Tescan Mira3 с автоэмиссионным катодом при ускоряющем напряжении 20 кВ. Для определения ориентировки отдельных зерен и анализа локальной текстуры использовали приставку EBSD HKL Inca с системой анализа Oxsford Instruments. Шаг сканирования - 0,1 мкм. Погрешность определения ориентации кристаллической решетки - не более $\pm 1^{\circ}$ (в среднем $\pm 0,6^{\circ}$ ). Малоугловые границы между локальными объемами строили на ориентационных картах при разориентациях от 1 до $15^{\circ}$ (линии толщиной в 1 пиксел), при разориентациях выше $15^{\circ}$ проводили высокоугловые границы (линии толщиной в 2 пикселя).

Анализ специальных границ между отдельными зернами осуществляли построением их на ориентационных картах с учетом заложенного в программное обеспечение стандартного критерия Брендона $\pm \Delta \Theta$. Для каждой границы он составляет конкретную величину: $\Delta \Theta=15^{\circ} /(\Sigma \mathrm{n})^{1 / 2}$, где $\Sigma \mathrm{n}-$ количество совпадающих узлов при наложении трехмерных кристаллических решеток.

\section{3. Методика обработки экспериментальных кривых}

Для анализа траекторий охлаждения использовали уравнение теплового баланса

$$
\frac{d Q(t)}{d t}=\Phi(t)-J(t)
$$

где $d Q(t)$ - фиксируемая величина тепловой энергии в образце в момент времени $t ; \Phi(t)-$ скорость выделения тепла в пластине при фазовом превращении; $J(t)$ - скорость отвода тепла с поверхности.

Поскольку в случае быстрого охлаждения определяющим является конвективный механизм отвода тепла, излучением Стефана-Больцмана можно пренебречь. В этом случае

$$
J(t)=-K\left(T(t)-T_{r}\right) S,
$$

где $T(t)$ - температура образца в момент времени $t$; $T_{r}$ - температура охлаждающего воздуха, $S$ - площадь поверхности образца; $K$ - коэффициент теплопередачи.

С учетом того, что изменение тепла в пластине определяется соотношением $\delta \mathrm{Q}=m \delta(c T)$, где $m$ - масса образца; $c$ - его удельная теплоемкость, уравнение (1) представляется в виде

$$
\frac{1}{V} \frac{d Q(t)}{d t}=\frac{d(\rho c T)}{d t}=\frac{1}{V} \Phi(t)-\frac{K}{h}\left(T-T_{r}\right)
$$

где $V$ - объем образца; $\rho$ - его плотность; $h$ - толщина пластины. Переходя к удельным величинам, запишем выражение (3) в форме

$$
\delta q(T(t)) \equiv \delta(\rho c T(t))=\phi(T(t)) \delta t-\frac{K}{h}\left(T-T_{r}\right) \delta t
$$

Последнее соотношение имеет простой физический смысл. При постоянных $\rho$ и $c$ изменение температуры $\delta T$ в процессе охлаждения за интервал времени $\delta t$ определяется наложением двух процессов: ее повышением вследствие тепловыделения при фазовом превраще- 
нии $\delta \varphi$ и понижением благодаря теплоотводу с поверхности со скоростью $K\left(T-T_{r}\right) / h$, не зависящей от фазового состояния и определяемой текущим значением температуры $T$.

Как видно из уравнения (3), при постоянных $\rho$ и $c$ величина $K\left(T-T_{r}\right) / h$ определяется скоростью изменения температуры $d T / d t$ в отсутствие превращения. То есть при $\varphi=0$ :

$$
\frac{\delta q_{1}}{\delta t} \equiv \rho c \frac{\delta T_{1}(t)}{\delta t}=-\frac{K}{h}\left(T_{1}(t)-T_{r}\right)
$$

С учетом выделяющейся в процессе превращения теплоты будем иметь

$$
\frac{\delta q}{\delta t} \equiv \rho c \frac{\delta T(t)}{\delta t}=\phi(t)-\frac{K}{h}\left(T(t)-T_{r}\right)
$$

Существенное уточнение: температура $T>T_{1}$. Из уравнений (6) и (5) получаем удельную теплоту, выделяющуюся к моменту времени $t$ :

$$
\frac{\delta \Delta q}{\delta t} \equiv \rho c \frac{\delta \Delta T(t)}{\delta t}=\phi(t)-\frac{K}{h} \Delta T(t)
$$

где $\delta q=q-q_{1} ; \Delta T=T-T_{1}$. Из уравнения (7) видно, что тепловой эффект полностью определяется фазовым превращением, если последним слагаемым можно пренебречь. В общем случае удельная теплота превращения, выделившаяся с начала превращения $t_{s}$ к моменту времени $t$, представлена выражением:

$$
Q(t)=\int_{t_{s}}^{t} \phi\left(t^{\prime}\right) d t^{\prime}=\int_{t_{s}}^{t}\left(\rho c \frac{\delta \Delta T\left(t^{\prime}\right)}{\delta t^{\prime}}+\frac{K}{h} \Delta T\left(t^{\prime}\right)\right) d t^{\prime}=\rho c \Delta T(t)+\frac{K}{h} \int_{t_{s}}^{t} \Delta T\left(t^{\prime}\right) d t^{\prime} .
$$

Таким образом, истинная теплота превращения $Q(t)$ больше чем кажущаяся $\rho c \Delta T(t)$ на величину $\frac{K}{h} \int_{t_{s}}^{t} \Delta T\left(t^{\prime}\right) d t^{\prime}$, определяемую теплоотводом с поверхности. Первое слагаемое полностью определяет теплоту превращения в отсутствие теплоотвода, когда температура постоянна до и после превращения, а ее изменение дает тепловой эффект. Величина $\Delta T$ с течением времени уменьшается и первое слагаемое стремиться к нулю, а тепловой эффект переходит во второе слагаемое, описывающее удаление тепловой энергии, и в предельном случае больших времен весь тепловой эффект определяется отведенным теплом.

Ограничимся интервалом времени фазового превращения $t_{f}-t_{s}\left(t_{f}-\right.$ время конца превращения), так что:

$$
Q(t)=\int_{t_{s}}^{t_{f}} \phi\left(t^{\prime}\right) d t^{\prime}=\rho c \Delta T\left(t_{f}\right)+\frac{K}{h} \int_{t_{s}}^{t_{f}} \Delta T\left(t^{\prime}\right) d t^{\prime}
$$

где первое слагаемое дает основной вклад, а второе - поправку на отведенную тепловую энергию.

Экспериментальные зависимости $T_{i}(t)$ как для высокотемпературной $(i \equiv \gamma)$, так и низкотемпературной $(i \equiv \alpha)$ областей на термограммах хорошо аппроксимируются выражениями:

$$
T(t)=T_{r}+\left(T_{i}+T_{r}\right) \exp \left(-\beta_{i} t\right)
$$

где $\beta_{i}-$ коэффициент $\left[\mathrm{c}^{-1}\right]$, определяемый в результате аппроксимации (рис. 2 б).

С учетом (10) уравнение (9) представляем в удобном для практического использования виде: 


$$
Q(t)=\rho c \Delta T\left(t_{f}\right)+\rho c \beta \int_{t_{s}}^{t_{f}} \Delta T\left(t^{\prime}\right) d t^{\prime}
$$

Алгоритм численного расчета тепловых эффектов с использованием метода конечных разностей приведен в [12]. При его проведении время протекания фазового превращения $\left(t_{f}-t_{s}\right)$ определяли из анализа зависимости $d T(t) / d t$ от $t$ (рис. 2 в). Теплоемкости $\gamma$ - и $\alpha$-фаз в [Дж / $\left.\left(\kappa^{\circ}{ }^{\circ} \mathrm{C}\right)\right]$, согласно [13], задавали выражениями: $C \gamma=0,1202 \mathrm{~T}+514,5 ; C \alpha=0,4832 \mathrm{~T}+434,6$. Долю превращения $(1-V \gamma /(V \gamma+V \alpha))$ находили нормированием участка функции $f(t)=\ln \left(T(t)-T_{r}\right)$, соответствующго времени протекания фазового превращения (рис. 2 г).

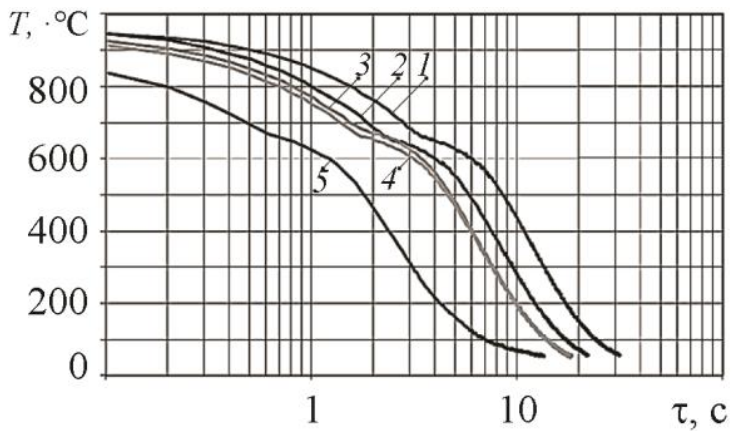

$a$

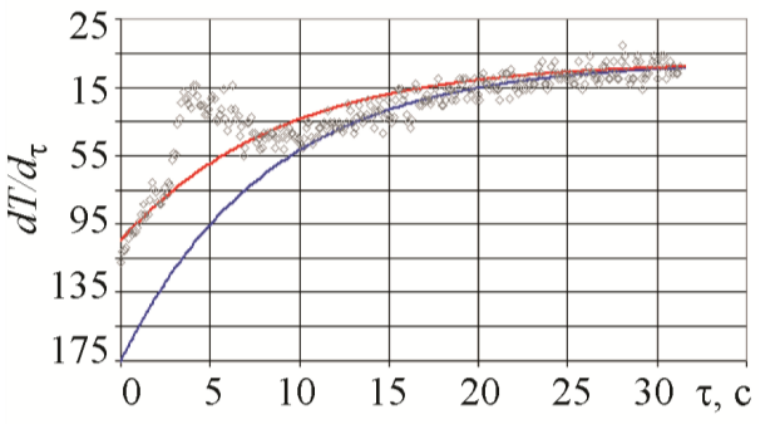

B

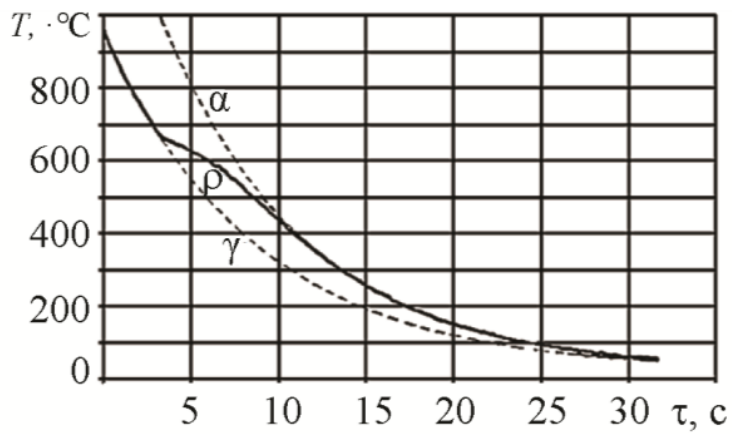

б

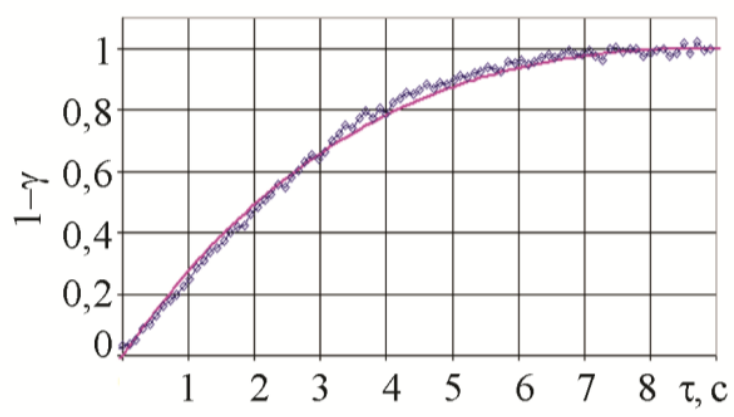

2

Рис. 2. Изменения температуры и производных от нее величин от времени при различных

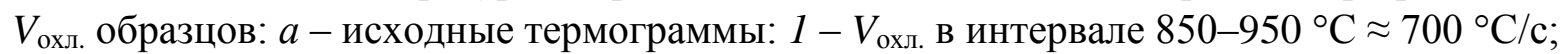
$2-\approx 450{ }^{\circ} \mathrm{C} / \mathrm{c} ; 3-\approx 200{ }^{\circ} \mathrm{C} / \mathrm{c} ; 4-\approx 190{ }^{\circ} \mathrm{C} / \mathrm{c} ; 5-\approx 170{ }^{\circ} \mathrm{C} / \mathrm{c} ; 6-\approx 160{ }^{\circ} \mathrm{C} / \mathrm{c} ; 7-\approx 110{ }^{\circ} \mathrm{C} / \mathrm{c}$; $\sigma$ - экспоненциальные аппроксимации высокотемпературной - $\gamma$ и низкотемпературной $\alpha$ частей термограммы $6 ;$ в - изменение $V_{\text {охл. }}$ образца 6 в процессе фазового превращения; 2 - изменение доли низкотемпературной фазы в процессе фазового превращения

\section{4. Результаты и обсуждение}

В случае высоких скоростей охлаждения $\left(V_{\text {охл. }} \geq 600{ }^{\circ} \mathrm{C} / \mathrm{c}\right)$ в образцах наблюдалась характерная для мартенсита пакетная структура, состоящая из чередующихся пластин толщиной от 0,5 до 2 мкм, длиной до 10 мкм (рис. 3 a). В пределах одного мартенситного пакета

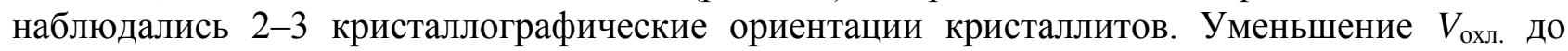
400-200 ${ }^{\circ} \mathrm{C} / \mathrm{c}$ привело к формированию заметно менее однородной структуры, в которой наряду с мартенситными пакетами фиксировались сравнительно более крупные, отдельные (внепакетные) кристаллиты, характеризующиеся неравновесными формами, имеющие максимальный размер около 20 мкм. 
При скоростях охлаждения $200-100{ }^{\circ} \mathrm{C} / \mathrm{c}$ в структуре практически исчезли мартенситные пакеты. Структура была представлена неравноосными кристаллитами неправильных геометрических форм максимальным размером до 20 мкм с мелкими темными включениями на их границах (рис. 3 б). При большем увеличении было видно, что включения являются не однородными выделениями, а характеризуются наличием собственной субструктуры, близкой к пакетной.

При дальнейшем уменьшении скорости охлаждения ниже $100{ }^{\circ} \mathrm{C} / \mathrm{c}$, формы кристаллитов основной структуры становились более равноосными. Границы кристаллитов имели тенденцию к выпрямлению. Возрастали как объемная доля, так и размеры темных включений. Ориентационная микроскопия позволяет утверждать, что включения являются мартенситными пакетами, в которых распад обогащенного углеродом аустенита реализовывался в последнюю очередь.

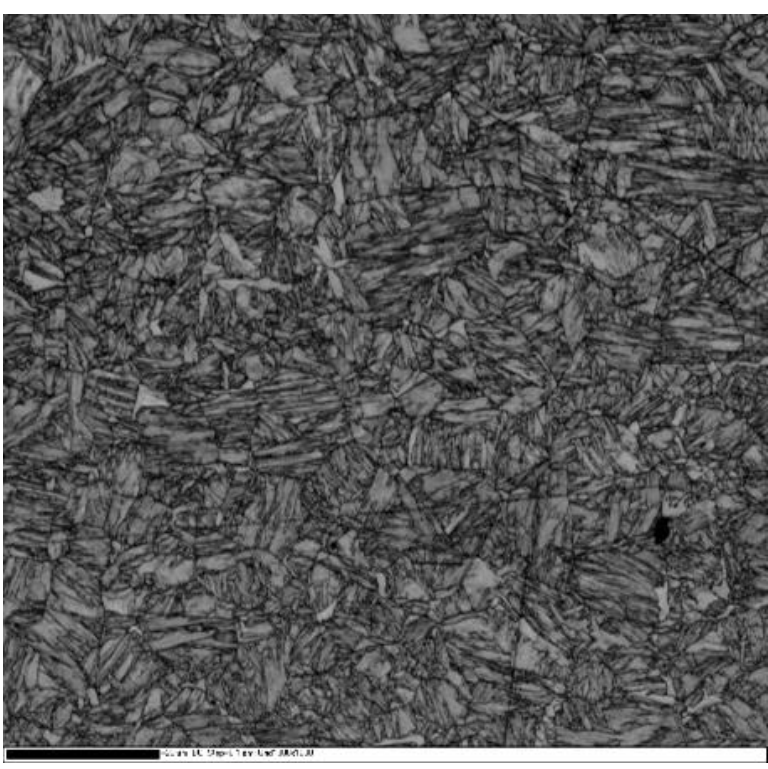

$a$

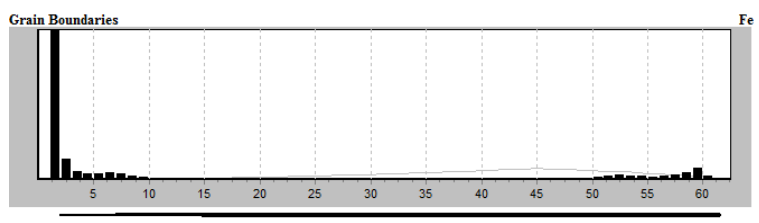

B

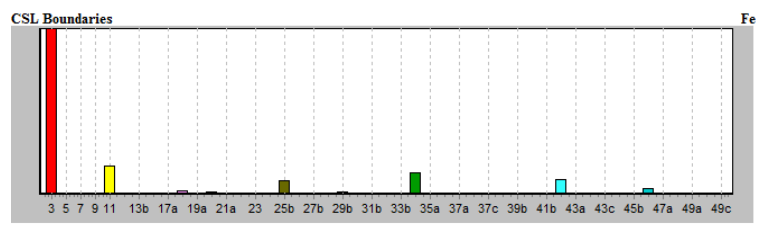

$\partial$

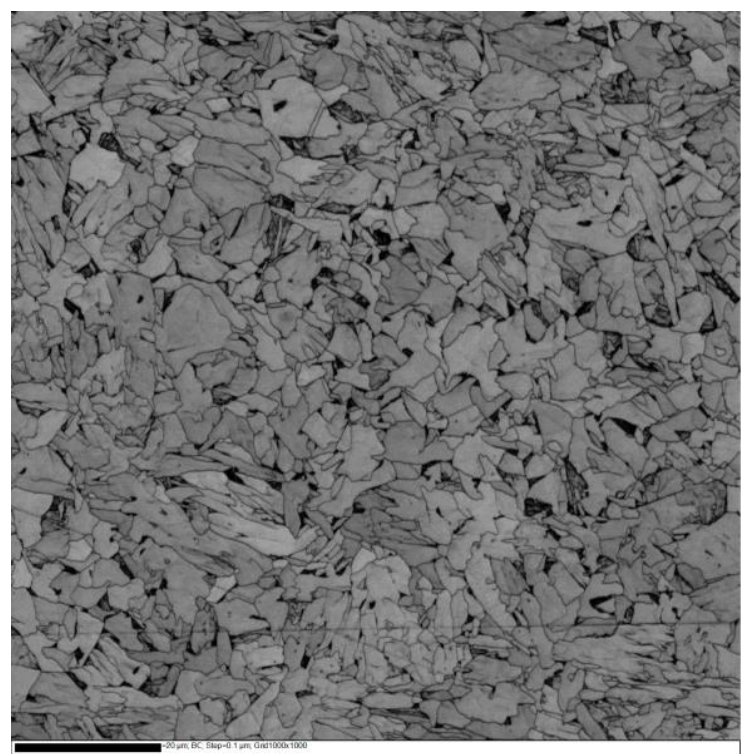

6

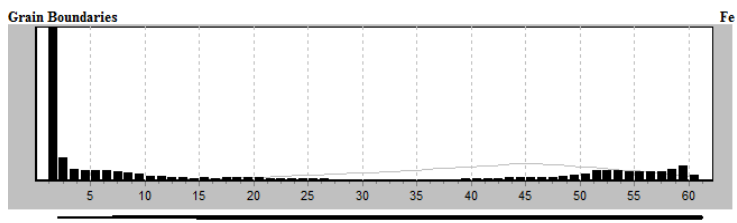

2

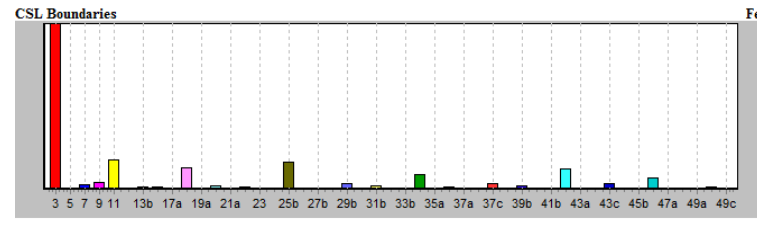

$e$

Рис. 3. Микроструктуры образцов, охлажденных с различными скоростями в обратно рассеянных электронах $(a, \sigma)$, и спектры межкристаллитных $(6,2)$ и $\operatorname{CSL}(\partial, e)$ границ; $a, 6, \partial-V_{\text {охл. }} \approx 700{ }^{\circ} \mathrm{C} / \mathrm{c} ; \sigma, z, e-V_{\text {охл. }} \approx 170{ }^{\circ} \mathrm{C} / \mathrm{c}$

Анализ спектров межкристаллитных границ (рис. 3 в-е) показывает, что все структуры, сформированные при скоростях охлаждения выше $100{ }^{\circ} \mathrm{C} / \mathrm{c}$, являются результатом сдвиго- 
вых фазовых превращений согласно [5, 11]: 1) среди межкристаллитных границ отсутствуют границы с углами разориентаций $\left.20-45^{\circ} ; 2\right)$ в спектре РСУ-границ наиболее выраженными являются $\Sigma 3, \Sigma 11, \Sigma 25 \mathrm{~b}, \Sigma 33 \mathrm{c}, \Sigma 41 \mathrm{c}$, что соответствует реализации фазовых превращений в соответствии с ориентационными соотношениями (OC), промежуточными между ОС Курдюмова-Закса и ОС Нишиямы-Вассермана. Таким образом, структуры, сформированные при скоростях охлаждения выше $600{ }^{\circ} \mathrm{C} / \mathrm{c}$, являются мартенситными. При скоростях $200-100{ }^{\circ} \mathrm{C} / \mathrm{c}$ формировались преимущественно (более чем на 95 \%) бейнитные структуры. Для данных диапазонов скоростей охлаждения определялись тепловые эффекты мартенситного и бейнитного превращений.

Рассчитанный по термограммам тепловой эффект бейнитного превращения составляет $120 \pm 10$ кДж/К. Его величина приблизительно в два раза превышает эффект мартенситного превращения $-50 \pm 20$ кДж/К. Последний результат хорошо согласуется с тепловым эффектом мартенситного превращения для низкоуглеродистых сталей как рассчитанного в известных исследованиях [14], так и измеренного в современных работах [15]. Отметим, что тепловой эффект бейнитного превращения оказался близким к энергии активации диффузии углерода в аустените низколегированных сталей [16]. Последнее позволяет утверждать, что кинетика протекания бейнитного превращения в основном определяется энергетикой наиболее медленного процесса, а именно, перераспределением атомов углерода в аустените, протекающим параллельно сдвиговой перестройке решеток ГЦК в ОЦК (ОЦТ).

\section{5. Заключение}

На оригинальном лабораторном стенде, позволяющем моделировать технологические параметры обработки горячекатаных листов в процессе ТМСР, для скоростей охлаждения $\geq 600$ и $200-100{ }^{\circ} \mathrm{C} / \mathrm{c}$ определены тепловые эффекты, соответственно, бейнитного $(120 \pm 10$ кДж/К) и мартенситного $(50 \pm 20$ кДж/К) фазовых превращений в трубной стали марки 06Г2МБ.

\section{Благодарность}

Работа выполнена на оборудовании ОАО «Аусферр в рамках государственного задания ФАНО России, тема «Структура» («Struсtиге»), номер госрегистрачии 0120146333, при поддержке Российского научного фонда, грант № 18-19-00085. Авторы выражают признательность за содействие программе поддержки ведущих университетов РФ в иелях повышения их конкурентоспособности № 211 Правительства РФ № 02.A03.21.0006. Aвторы благодарят ПАО «ММК» за поддержку и содействие в организаџии исследования.

\section{Литература}

1. Effects of carbon equivalent and cooling rate on tensile and Charpy impact properties of highstrength bainitic steels / H. K. Sung, S. Y. Shin, B. Hwang, C. G. Lee, N. J. Kim, S. Lee // Materials Science and Engineering A. - 2011. - Vol. 530. - P. 530-538. - DOI: 10.1016/j.msea.2011.10.015.

2. The thermomechanical controlled processing of high-strength steel plate: A New View of toughness based on modern metallography / X. J. Liang, M. J. Hua, C. I. Garcia, A. J. Deardo // Materials Science Forum. - 2013. - Vol. 762. - P. 38-46. - DOI: 10.4028/www.scientific.net/MSF.762.38.

3. Goli-Oglu E. A., Éfron L. I., Morozov Yu. D. Effect of deformation regime in main stages of controlled rolling on pipe steel microstructure // Metal Science and Heat Treatment. - 2013. Vol. 55. - P. 294-297. - DOI: 10.1007/s11041-013-9622-8.

4. Shigeru E., Naoki N. Development of Thermo-Mechanical Control Process (TMCP) and high performance steel in JFE Steel // JFE Technical Report. - 2015. - No. 20. - P. 1-7.

5. Texture Inheritance on Phase Transition in Low-Carbon, Low-Alloy Pipe Steel after Thermomechanical Controlled Processing / M. L. Lobanov, M. D. Borodina, S. V. Danilov, 
I. Y. Pyshmintsev, A. O. Struin // Steel in Translation. - 2017. - Vol. 47. - P. 710-716. DOI: $10.3103 / \mathrm{S} 0967091217110055$.

6. Effect of bainite crystallographic texture on failure of pipe steel sheets made by controlled thermomechanical treatment / I. Y. Pyshmintsev, A. O. Struin, A. M. Gervasyev, M. L. Lobanov, G. M. Rusakov, S. V. Danilov, A. B. Arabey // Metallurgist. - 2016. - Vol. 60. - P. 405-412. DOI: $10.1007 / \mathrm{s} 11015-016-0306-7$.

7. Microstructural design in quenched and partitioned (Q\&P) steels to improve their fracture properties / I. Sabirov, I. de Diego-Calderón, J. M. Molina-Aldareguia, C. Föjer, R. Thiessen, R. H. Petrov // Materials Science and Engineering A. - 2016. - Vol. 657. - P. 136-146. DOI: 10.1016/j.msea.2016.01.011.

8. Effects of ausforming temperature on bainite transformation, microstructure and variant selection in nanobainite steel / W. Gong, Y. Tomota, A. M. Paradowska, J. F. Kelleher, S. Y. Zhang // Acta Materialia. - 2013. - Vol. 61. - P. 4142-4154. - DOI: 10.1016/j.actamat.2013.03.041.

9. Х Хулка К., Петерс П., Хайстеркамп Ф. Тенденции разработки сталей для труб большого диаметра // Сталь. - 1997. - № 10. - С. 62-67.

10. Методы исследования текстур в материалах: учебное пособие / М. Л. Лобанов, А. С. Юровских, Н. И. Кардонина, Г. М. Русаков. - Екатеринбург : Изд-во Урал. ун-та, 2014. -115 c.

11. Investigation of Special Misorientations in Lath Martensite of Low-Carbon Steel Using the Method of Orientation Microscopy / M. L. Lobanov, G. M. Rusakov, A. A. Redikul'tsev, S. V. Belikov, M. S. Karabanalov, E. R. Struina, A. M. Gervas'ev // The Physics of Metals and Metallography. - 2016. - Vol. 117. - P. 254-259. - DOI: 10.1134/S0031918X1603008X.

12. Thermal effect of bainitic transformation in tube steels during accelerated cooling / M. L. Lobanov, G. M. Rusakov, V. N. Urtsev, M. L. Krasnov, E. D. Mokshin, A. V. Shmakov, S. I. Platov // Letters on Materials. - 2018. - Vol. 8 (3). - P. 246-251. - DOI: 10.22226/2410-35352018-3-246-251.

13. Иванов Д. А., Куваев Н. В., Куваева Т. В. Расчет теплоемкости низкоуглеродистой низколегированной стали при моделировании неизотермических фазовых превращений // Теория и практика металлургии. - 2010. - № 1-2. - С. 43-48.

14. Суфиянова Г. И., Спивак Л. В. Фазовые превращения низкоуглеродистых мартенситных сталях // Физика для пермского края : материалы региональной научно-практической конференции студентов, аспирантов и молодых ученых / под общ. ред. Н. Н. Картавых. Пермский государственный национальный исследовательский университет, 2016 - С. 37-39. 15. Bhadeshia H. K. D. H. Martensite and bainite in steels: Transformation mechanism \& mechanical properties // Journal De Physique. IV : JP. - 1997. - Vol. 7 (5). - P. C5-367-C5-376.

16. Гольдштейн М. И., Попов В. В. Растворимость фаз внедрения при термической обработке стали. М : - Металлургия, 1989. - 200 с. 